\title{
PARTIAL LINEARIZATION WITHOUT LINESEARCH FOR NETWORK EQUILIBRIUM PROBLEMS
}

\author{
Igor Konnov $^{1}$, Erkki Laitinen ${ }^{2}$ and Olga Pinyagina ${ }^{3}$ \\ ${ }^{1}$ Kazan Federal University, Department of System Analysis and Information Technologies, 420008 Kazan, Russia \\ ${ }^{2}$ University of Oulu, Unit of Mathematical Sciences, 90014 Oulu, Finland \\ ${ }^{3}$ Kazan Federal University, Department of Data Mining and Operations Research, 420008 Kazan, Russia
}

\begin{abstract}
In the present paper, we propose a simplified variant of the partial linearization method for the network equilibrium problem. This modification uses an adaptive stepsize procedure. It maintains the basic convergence properties and enables one to reduce the total volume of computational efforts. The key element of this approach is a given majorant stepsize sequence converging to zero. In accordance with this majorant, the next decreased value of the stepsize is taken only when the current iterate does not give a sufficient descent, which is estimated with the help of an Armijo-type condition. Preliminary numeric tests show the efficiency of this approach in comparison with the original variant of method.
\end{abstract}

\section{KEYWORDS}

Network Equilibrium Problem, Partial Linearization Method, Adaptive Stepsize Choice

\section{INTRODUCTION}

Network equilibrium problems arise in different areas, including telecommunication and transportation networks. They have long been known and investigated in detail, for example, see (Dafermos1980; Dafermos 1982; Nagurney 1999; Patriksson 2015). The simple feasible sets and special decomposable structure of these problems allow one to use modifications of the conditional gradient or partial linearization methods, where the descent direction can easily be found without complex iterative procedures (Konnov et al 2016). At the same time, these problems usually have the great dimension, and it is desirable to develop solution methods which also reduce the total volume of computations in stepsize search. In the present paper, we propose a version of the partial linearization method which uses an adaptive procedure for stepsize choice formulated initially for the conditional gradient method in (Konnov 2018a; Konnov 2018b). The key element of this approach is a given majorant stepsize sequence converging to zero. In accordance with this majorant, the next decreased value of the stepsize is taken only when the current iterate does not give a sufficient descent, which is estimated with the help of an Armijo-type condition. Preliminary numeric tests show the efficiency of this approach in comparison with the original variant of method.

\section{PARTIAL LINEARIZATION METHOD}

Let us remind the general scheme of the partial linearization method. The partial linearization approach was proposed in (Mine et al 1981) for optimization problems and developed in (Patriksson 2015) for variational inequalities (VI for short). This approach has advantages when the feasible set of the considered problem has a relatively simple structure and the objective function can be decomposed into two parts, where one of them is suitable for linearization, and the other is sufficiently simple. We consider the following optimization problem

$$
\min _{x \in D} \rightarrow \mu(x)
$$

where the objective function $\mu: \mathbb{R}^{n} \rightarrow \mathbb{R}$ is the sum of two functions $\mu(x)=f(x)+h(x)$, where the function $f$ is smooth, the function $h$ is convex, and the feasible domain $D$ is a convex closed bounded set in $\mathbb{R}^{n}$. 
We now describe the partial linearization method for problem (1). Let us given a point $x^{k} \in D$ at the $k$ th iteration, $k=1,2, \ldots$. Find $z^{k} \in D$ as a solution to the auxiliary problem

$\min _{x \in D} \rightarrow\left\langle f^{\prime}\left(x^{k}\right), x\right\rangle+h(z)$,
set $d^{k}=z^{k}-x^{k}$ and define the next iterate $x^{k+1}=x^{k}+\lambda_{k} d^{k}$, where the step $\lambda_{k}$ can be found with a suitable exact or inexact procedure for the one-dimensional minimization problem. This method converges to a stationary point of problem (1).

As we noted above, the original partial linearization method usually involves a certain exact or inexact stepsize linesearch procedure. Recently in papers (Konnov 2018a; Konnov 2018b) a general scheme of simple adaptive stepsize choice was proposed for conditional gradient type methods. This scheme takes into account the behavior of the problem along the main iterative sequence. In the present paper, we apply this approach to the partial linearization method for network equilibrium problems.

\section{NETWORK EQUILIBRIUM PROBLEMS}

Let us recall the formulation of the network equilibrium problem with elastic demand, which was originally given in (Dafermos 1982). It describes a model of traffic or information data flows.

Let $V$ be a set of network nodes, $A$ be a set of directed arcs (links). In addition, a set $W$ of origin-destination (O/D) pairs $(i, j), i, j \in V$ is given. For each O/D-pair $w \in W$ a set of paths $P_{w}$ is given; each path presents a simple chain of arcs starting at the origin node and ending at the destination node of O/D-pair. We denote by $x_{p}$ a variable flow value passing along path $p$, for all $p \in P_{w}, w \in W$.

Additionally, for each O/D-pair $w \in W$ a demand variable $y_{w}$ is given. It presents a flow outgoing from the origin and ingoing to the destination. We assume these variables to be bounded from below and above with boundaries $0 \leq \hat{\gamma}_{w}<\breve{\gamma}_{w}$.

The network equilibrium problem is to find a distribution of the required demands for all O/D pairs among the sets of paths by using a certain (equilibrium) criterion.

The feasible set has the form:

$$
U=\left\{(x, y) \mid \sum_{p \in P_{w}} x_{p}=y_{w}, x_{p} \geq 0, p \in P_{w}, y_{w} \in\left[\hat{\gamma}_{w}, \breve{\gamma}_{w}\right], w \in W\right\} .
$$

The correspondence of paths and arcs is given by the incidence matrix with elements

$$
\alpha_{p a}= \begin{cases}1, \text { if } \operatorname{arc} a \text { belongs to path } p ; \\ 0, & \text { otherwise. }\end{cases}
$$

Then the arc flow value is defined as the sum of the corresponding path flows, for each arc $a \in A$ :

$$
f_{a}=\sum_{w \in W} \sum_{p \in P_{w}} \alpha_{p a} x_{p} \text {. }
$$

Let a continuous cost function $c_{a}$ be given for each $a \in A$; it can depend on all the arc flows in general. In addition, a so-called disutility continuous function $h_{w}$ is given for each O/D pair $w \in W$. In the general case, the disutility functions can depend on the whole demand vector $y$.

The path cost function is defined as follows:

$$
g_{p}(x)=\sum_{a \in A} \alpha_{p a} c_{a}(f),
$$

for each path $p$, where $f$ is the vector of arc flows $f_{a}, a \in A$.

We denote by $G$ and $H$ the vectors with the components $g_{p}, p \in P_{w}, w \in W$, and $h_{w}, w \in W$, respectively.

For finding an equilibrium state of this network, one can solve the following variational inequality: find an element $\left(x^{*}, y^{*}\right) \in U$ such that

$$
\left\langle G\left(x^{*}\right), x-x^{*}\right\rangle-\left\langle H\left(y^{*}\right), y-y^{*}\right\rangle \geq 0 \quad \forall(x, y) \in U .
$$

In paper (Konnov 2015), it was shown that the above network equilibrium problem can be treated as a two-side multicommodity market equilibrium problem. The optimality conditions for this network equilibrium problem are following. that

A point $\left(x^{*}, y^{*}\right) \in U$ constitutes a network equilibrium, if for each $w \in W$ there exists a number $\lambda_{w}$ such

$$
g_{p}\left(x^{*}\right)\left\{\begin{array}{ll}
\geq \lambda_{w} & \text { if } x_{p}^{*}=0, \\
=\lambda_{w} & \text { if } x_{p}^{*}>0,
\end{array} \quad \forall p \in P_{w},\right.
$$




$$
h_{w}\left(y^{*}\right) \begin{cases}\leq \lambda_{w} & \text { if } y_{w}^{*}=\hat{\gamma}_{w}, \\ =\lambda_{w} & \text { if } y_{w}^{*} \in\left(\hat{\gamma}_{w}, \breve{\gamma}_{w}\right), \\ \geq \lambda_{w} & \text { if } y_{w}^{*}=\breve{\gamma}_{w} .\end{cases}
$$

The above problem formulation generalizes the classical network equilibrium problems with fixed and elastic demands (see also (Pinyagina 2017), where it was called the network equilibrium problem with mixed demand). If, for example, we choose cost and disutility functions such that $g_{p}(x)>h_{w}(y)$ for all $p \in P_{w}, w \in$ $W,(x, y) \in U$, then we obtain the network equilibrium problem with fixed demand, where the vector of fixed demand is given by components $\hat{\gamma}_{w}, w \in W$. On the other hand, in the classical network equilibrium problem with elastic demand $\hat{\gamma}_{w}=0$ and $\breve{\gamma}_{w}=\infty$.

In what follows, we assume that each arc cost function $c_{a}$ depends on $f_{a}$ only, $\forall a \in A$, each disutility function $h_{w}$ depends on $y_{w}$ only, $\forall w \in W$. Then the mappings $G$ and $H$ are potential, and there exist the functions

$$
\mu_{a}\left(f_{a}\right)=\int_{0}^{f_{a}} c_{a}(t) d t \quad \forall a \in A, \quad \sigma_{w}\left(y_{w}\right)=\int_{0}^{y_{w}} h_{w}(t) d t \quad \forall w \in W
$$

In this case, VI (3) presents the optimality condition for the following optimization problem:

$$
\min _{u \in U} \rightarrow \psi(u),
$$

where $u=(x, y)$,

$$
\psi(x, y)=\left\{\sum_{a \in A} \mu_{a}\left(f_{a}\right)-\sum_{w \in W} \sigma_{w}\left(y_{w}\right)\right\},
$$

$f_{a}, \forall a \in A$ are defined in (2). Therefore, each solution to problem (4) solves problem (3). The reverse assertion is true, if, for example, the mappings $G$ and $-H$ are monotone.

We note that the feasible set $U$ of the network equilibrium problem (3) is the Cartesian product of simplex-type sets. In addition, the potentiality of mappings $G$ and $H$ provides decomposition properties of the network equilibrium problem, which we will use in the following section.

\section{PARTIAL LINEARIZATION METHOD WITHOUT LINESEARCH}

In (Konnov et al 2016), the partial linearization approach was applied to the network equilibrium problem with elastic demand. We suppose that $h_{w}(y)=h_{w}\left(y_{w}\right), \forall w \in W$.

The auxiliary direction finding problem is described as follows (Konnov et al, 2016). At the $k$ th iteration $(k=0,1, \ldots)$ of the main process we have the vector of path flows $x^{k}$. We calculate the values of cost functions $g_{p}\left(x^{k}\right)$, for all $p \in P_{w}, w \in W$. Then we intend to find a vector $\left(\bar{x}^{k}, \bar{y}^{k}\right) \in W$ which is a solution to the auxiliary linearized VI:

$$
\sum_{w \in W}\left[\sum_{p \in P_{w}} g_{p}\left(x^{k}\right)\left(x_{p}-\bar{x}_{p}^{k}\right)-h_{w}\left(\bar{y}^{k}\right)\left(y_{w}-\bar{y}_{w}^{k}\right)\right] \geq 0 \quad \forall(x, y) \in U,
$$

or to the equivalent optimization problem

$$
\min _{(x, y) \in U} \rightarrow \sum_{w \in W}\left[\sum_{p \in P_{w}} g_{p}\left(x^{k}\right) x_{p}-\sigma_{w}\left(y_{w}\right)\right],
$$

where $h_{w}\left(y_{w}\right)=\sigma^{\prime}{ }_{w}\left(y_{w}\right)$. In what follow we assume that the functions $\sigma_{w}\left(y_{w}\right)$ are convex, for all $w \in W$. The above stated problems can be decomposed into a family of independent problems, per one problem for each O/D pair. Hence the algorithm has the following simple scheme (Konnov et al 2016).

\section{Algorithm A}

For certain O/D pair $w \in W$, we calculate the set of shortest paths $\bar{P}_{w}^{k}$ with costs values $g_{p}\left(x^{k}\right)$. Let $\tilde{\lambda}_{w}=g_{p}\left(x^{k}\right), \forall p \in \bar{P}_{w}^{k}$. Hence the following three cases are possible.

1) If $h_{w}\left(\hat{\gamma}_{w}\right) \leq \tilde{\lambda}_{w}$, then set $\bar{y}_{w}^{k}=\hat{\gamma}_{w}$.

2) If $h_{w}\left(\breve{\gamma}_{w}\right) \geq \tilde{\lambda}_{w}$, then set $\bar{y}_{w}^{k}=\breve{\gamma}_{w}$.

3) Otherwise we have $h_{w}\left(\breve{\gamma}_{w}\right)<\tilde{\lambda}_{w}<h_{w}\left(\hat{\gamma}_{w}\right)$, then find the value of demand $\bar{y}_{w}^{k} \in\left[\hat{\gamma}_{w}, \breve{\gamma}_{w}\right]$ such that $h_{w}\left(\bar{y}_{w}^{k}\right)=\tilde{\lambda}_{w}$.

Distribute the demand value $\bar{y}_{w}^{k}$ among paths $p \in \bar{P}_{w}^{k}$ (it is possible to associate the whole demand with one path). Set $\bar{x}_{p}^{k}=0 \forall p \in P_{w} \backslash \bar{P}_{w}^{k}$.

Following this scheme, one can find an exact solution to problem (5) or (6) and obtain a descent direction for the main problem. 
In the present section, we propose a variant of the partial linearization method, in which the stepsize choice is realized without iterative procedures.

\section{Partial linearization method without linesearch (PLWLS)}

Step 0 . Choose an initial point $u^{0} \in U$, a coefficient $\beta \in(0,1)$, and a majorant sequence $\left\{\tau_{l}\right\} \rightarrow 0, \tau_{l} \in$ $(0,1)$. Set $k=0, l=0$, choose an initial step size $\lambda_{0} \in\left(0, \tau_{0}\right]$.

Step 1. Sequentially applying Algorithm A for all $w \in W$, find a point $\bar{u}^{k}=\left(\bar{x}^{k}, \bar{y}^{k}\right)$.

Step 2. If $\left\langle\psi^{\prime}\left(u^{k}\right), \bar{u}^{k}-u^{k}\right\rangle=0$, then stop. Otherwise set $d^{k}=\bar{u}^{k}-u^{k}$ and $u^{k+1}=u^{k}+\lambda_{k} d^{k}$. Step 3. If

$$
\psi\left(u^{k+1}\right)-\psi\left(u^{k}\right) \leq \beta \lambda_{k}\left\langle\psi^{\prime}\left(u^{k}\right), d^{k}\right\rangle,
$$

then take $\lambda_{k+1} \in\left[\lambda_{k}, \tau_{l}\right]$. Otherwise set $\lambda_{k+1}^{\prime}=\min \left\{\lambda_{k}, \tau_{l+1}\right\}, l=l+1$, and take $\lambda_{k+1} \in\left(0, \lambda_{k+1}^{\prime}\right]$. Step 4. Set $k=k+1$ and go to Step 1 .

We denote by $U^{0}$ the solution set of problem (3), by $U^{*}$ the solution set of problem (4), and by $Z(u)$ the solution set of problem (5) and (6) at point $u \in U$.

The next lemmas generalize the main properties of the partial linearization method and follow Lemma 3.1-3.3 from (Mine et al 1981).

Lemma 1 For any $u \in U$ and $z \in Z(u)$ we have $\left\langle\psi^{\prime}(u), z-u\right\rangle \leq 0$.

Lemma 2 The mapping $u \mapsto Z(u)$ is closed for all $u \in U$.

Lemma 3 The following assertions are equivalent:

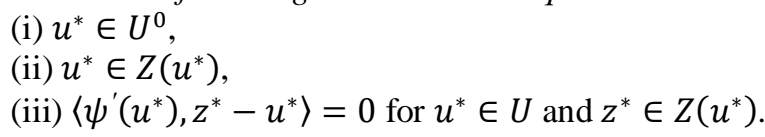

Let us prove the convergence of the proposed method. If the method stops at Step 2 at some point $u^{k}$, then $u^{k}$ solves VI (3) in view of Lemma 3. If in addition the function $\psi$ is convex, it is also a solution to optimization problem (4).

In what follows, we assume that the iterative sequence $\left\{u^{k}\right\}$ is infinite.

Theorem 1 (i) The sequence $\left\{u^{k}\right\}$ generated by PLWLS has a limit point, which is a solution to VI (3).

(ii) If in addition the function $\psi$ is convex, then all the limit points of the sequence $\left\{u^{k}\right\}$ are solutions to optimization problem (4).

Proof. We note that the iterative sequences $\left\{u^{k}\right\}$ and $\left\{\bar{u}^{k}\right\}$ are contained in a bounded set $U$, therefore they have limit points. Then the two cases are possible.

Case 1. The number of changes of the index $l$ is finite.

Then we have $\lambda_{k} \geq \bar{\lambda}>0$ for numbers $k$ large enough, therefore we obtain from condition (7)

$$
\psi\left(u^{k+1}\right) \leq \psi\left(u^{k}\right)-\beta \lambda_{k}\left\langle\psi^{\prime}\left(u^{k}\right), d^{k}\right\rangle \leq \psi\left(u^{k}\right)-\beta \bar{\lambda}\left\langle\psi^{\prime}\left(u^{k}\right), d^{k}\right\rangle
$$

for $k$ large enough. Since $\psi\left(u^{k}\right) \geq \psi^{*}>-\infty$, we obtain

$$
\lim _{k \rightarrow \infty}\left\langle\psi^{\prime}\left(u^{k}\right), d^{k}\right\rangle=0 .
$$

Let $u^{\prime}$ be an arbitrary limit point of the sequence $\left\{u^{k}\right\}$. Taking a subsequence, if necessary, we take the corresponding limit point $u^{\prime \prime}$ of the sequence $\left\{\bar{u}^{k}\right\}$, i.e.,

Hence we obtain

$$
u^{\prime}=\lim _{s \rightarrow \infty} u^{k_{l}}, u^{\prime \prime}=\lim _{s \rightarrow \infty} \bar{u}^{k_{l}}
$$

$$
\lim _{k \rightarrow \infty}\left\langle\psi^{\prime}\left(u^{\prime}\right), u^{\prime \prime}-u^{\prime}\right\rangle=0,
$$

but by Lemma 2, the mapping $x \mapsto Z(x)$ is closed, therefore $u^{\prime \prime} \in Z\left(u^{\prime}\right)$. From Lemma 3 we have that $u^{\prime} \in U^{0}$. Hence all the limit points of the iterative sequence $\left\{u^{k}\right\}$ belong to the set $U^{0}$, and assertion (i) was proven in this case.

Case 2: The number of changes of the index l is infinite. 
In this case, there exists an infinite subsequence of indices $\left\{k_{l}\right\}$ such that $u^{k_{l}+1}=u^{k_{l}}+\lambda_{k_{l}} d^{k_{l}}$ and condition (7) is violated:

In addition,

$$
\psi\left(u^{k_{l}}+\lambda_{k_{l}} d^{k_{l}}\right)-\psi\left(u^{k_{l}}\right)=\psi\left(u^{k_{l}+1}\right)-\psi\left(u^{k_{l}}\right)>-\beta \lambda_{k_{l}}\left\langle\psi^{\prime}\left(u^{k_{l}}\right), d^{k_{l}}\right\rangle .
$$

$$
\lambda_{k_{l}} \in\left(0, \tau_{l}\right], \quad \lambda_{k_{l}+1} \in\left(0, \tau_{l+1}\right]
$$

and $\lim _{l \rightarrow \infty} \tau_{l}=0$. Therefore, $\lim _{l \rightarrow \infty} \lambda_{k_{l}}=0$. Note that since the subsequence $\left\{d^{k_{l}}\right\}$ is bounded, the limit points of the subsequences $\left\{u^{k_{l}+1}\right\}$ and $\left\{u^{k_{l}}\right\}$ coincide. Let us take an arbitrary limit point $u^{\prime}$ of this subsequence $\left\{u^{k_{l}}\right\}$. Without loss of generality we can assume that

$$
u^{\prime}=\lim _{s \rightarrow \infty} u^{k_{l}}, u^{\prime \prime}=\lim _{s \rightarrow \infty} \bar{u}^{k_{l}} \text {. }
$$

Since $\lambda_{k_{l}} \rightarrow 0$ with $l \rightarrow \infty$, taking the limit as $k=k_{l} \rightarrow \infty$ in the correlation

$$
\left(\lambda_{k_{l}}\right)^{-1}\left[\psi\left(u^{k_{l}}+\lambda_{k_{l}} d^{k_{l}}\right)-\psi\left(u^{k_{l}}\right)\right]>\beta\left\langle\psi^{\prime}\left(u^{k_{l}}\right), d^{k_{l}}\right\rangle
$$

we obtain $\left\langle\psi^{\prime}\left(u^{\prime}\right), u^{\prime \prime}-u^{\prime}\right\rangle \geq \beta\left\langle\psi^{\prime}\left(u^{\prime}\right), u^{\prime \prime}-u^{\prime}\right\rangle$, i.e., $\left\langle\psi^{\prime}\left(u^{\prime}\right), u^{\prime \prime}-u^{\prime}\right\rangle \geq 0$. Hence by Lemmas $1-3$ it follows that $u^{\prime} \in U^{0}$. So, all the limit point of the subsequences $\left\{u^{k^{l}+1}\right\}$ and $\left\{u^{k_{l}}\right\}$ belong to the set $U^{0}$. Assertion (i) was also proven for this case.

Now we assume that the solution sets of problems (3) and (4) coincide, i.e., $U^{*}=U^{0}$. In Case 1, evidently, assertion (i) holds true.

In Case 2, as we showed above, the limit points of the subsequences $\left\{u^{k_{l}}\right\}$ and $\left\{u^{k_{l}+1}\right\}$ coincide and now all they belong to the set $U^{*}$. For any index $k$ we define the index $m(k)$ as follows

$$
m(k)=\max \left\{j: j \leq k, \psi\left(u^{j}\right)-\psi\left(u^{j-1}\right)>-\beta \lambda_{j-1}\left\|d^{j-1}\right\|^{2}\right\},
$$

i.e., $m(k)$ is the closest to $k$ but not greater index from the subsequence $\left\{u^{k_{l}+1}\right\}$. This means that $m(k)=k$ if $\psi\left(u^{k}\right)-\psi\left(u^{k-1}\right)>-\beta \lambda_{k-1}\left\|d^{k-1}\right\|^{2}$. By definition, we have

$$
\psi\left(x^{k}\right) \leq \psi\left(x^{m(k)}\right) \text {. }
$$

Now let us take an arbitrary limit point $u^{*}$ of the sequence $\left\{u^{k}\right\}$, i.e., $\lim _{s \rightarrow \infty} u^{t_{s}}=u^{*}$. Then we construct the corresponding infinite subsequence $\left\{u^{m\left(t_{s}\right)}\right\}$. From condition (8) we have $\psi^{*} \leq \psi\left(u^{t_{s}}\right) \leq \psi\left(u^{m\left(t_{s}\right)}\right)$, but all the limit points of the sequence $\left\{u^{m\left(t_{s}\right)}\right\}$ belong to the set $U^{*}$ because it is contained in the sequence $\left\{u^{k_{S}+1}\right\}$. Choose any limit point $u^{\prime}$ of $\left\{u^{m\left(t_{s}\right)}\right\}$. Then, taking a subsequence if necessary, we obtain

$$
\psi^{*} \leq \psi\left(u^{*}\right) \leq \psi\left(u^{\prime}\right)=\psi^{*},
$$

therefore $u^{*} \in U^{*}$. This means that all the limit points of the iterative sequence $\left\{u^{k}\right\}$ belong to the set $U^{*}$. We conclude that assertion (ii) also holds true. The proof is complete.

In the next section, we describe the results of preliminary numerical tests, which show the efficiency of the above proposed modification of the partial linearization method without linesearch.

\section{PRELIMINARY TEST CALCULATIONS}

We compared the proposed modification of the partial lineariation method without linesearch (PLWLS) and its original version with inexact linesearch (PLI) (Konnov et al 2016). The stopping criterion has the form $\left\langle\psi^{\prime}\left(u^{k}\right), u^{k}-z^{k}\right\rangle \leq \Delta$ for given $\Delta>0$, where $z^{k}$ is a solution to the problem

$$
\min _{z \in U} \rightarrow\left\langle\psi^{\prime}\left(u^{k}\right), z\right\rangle \text {. }
$$

The parameters of methods are $\theta=0.5, \beta=0.5, \tau_{0}=1, \tau_{l+1}=0.9 \tau_{l}$

Example 1. We took the test network example from (Bertsekas et al 1982), but we supposed that all arcs are bypass (Figure 1).

The cost functions are $c_{a}\left(f_{a}\right)=1+0.5 f_{a}, \forall a \in A$. The disutility functions are $h_{w}\left(y_{w}\right)=30-0.5 y_{w}$, $\forall w \in W$. The lower boundaries of demand $\hat{\gamma}_{w}$ are $(2,4,6,8,10)$, and all the upper boundaries $\breve{\gamma}_{w}, w \in W$ are equal to 50. The calculation results for Example 1 are presented in Table 1. 


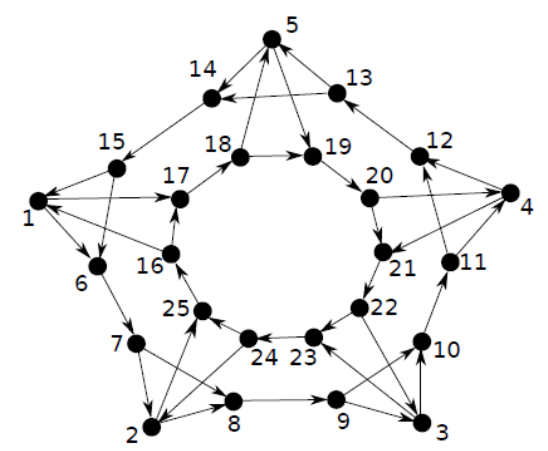

Figure 1. Network 1, Bertsekas-Gafni network, 25 nodes, 5 O/D pairs: (1-4), (2-5), (3-1), (4-2), (5-3)

Table 1. Example 1, numbers of iterations and calculation time

\begin{tabular}{rrrrr}
\hline \multicolumn{3}{c}{ PLWLS } & \multicolumn{2}{c}{ PLI } \\
\hline$\Delta$ & iterations & time (s) & iterations & time (s) \\
\hline 0.2 & 2233 & 0.062 & 2551 & 0.156 \\
0.1 & 8519 & 0.140 & 5747 & 0.312 \\
0.05 & 24132 & 0.359 & 11644 & 0.639 \\
\hline
\end{tabular}

Example 2. Network 2 (Figure 2) is a slightly modified network from (Nagurney 1984). The vector of $\hat{\gamma}_{w}, w \in W$ is $(5,5,5)$. Other parameters have the same values as in Example 1. The calculation results for this network are presented in Table 2.

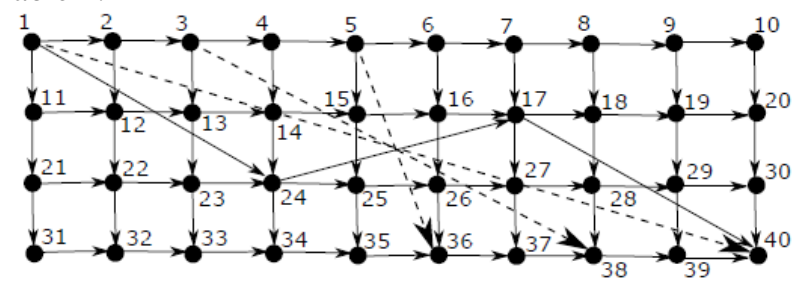

Figure 2. Network 2, Nagurney network, 40 nodes, 3 O/D pairs: (1-40), (3-38), (5-36)

Table 2. Example 2, numbers of iterations and calculation time

\begin{tabular}{rrrrr}
\hline \multicolumn{3}{c}{ PLWLS } & \multicolumn{2}{c}{ PLI } \\
\hline$\Delta$ & iterations & time (s) & iterations & time (s) \\
\hline 0.2 & 4199 & 0.156 & 4272 & 0.484 \\
0.1 & 14375 & 0.406 & 8418 & 0.920 \\
0.05 & 39867 & 1.076 & 17575 & 2.012 \\
\hline
\end{tabular}

Example 3. In conclusion of this section, we consider the third randomly-generated network containing 20 nodes, 108 arcs, and 20 OD-pairs. The values $\breve{\gamma}_{w}$ equal 20 for all,$w \in W$. Other parameters have the same values as in Example 1. The calculation result for this network is presented in Table 3.

Table 3. Example 3, numbers of iterations and calculation time

\begin{tabular}{rrrrr}
\hline \multicolumn{3}{c}{ PLWLS } & \multicolumn{2}{c}{ PLI } \\
\hline$\Delta$ & iterations & time (s) & iterations & time (s) \\
0.2 & 71013 & 3.082 & 19078 & 4.539 \\
0.1 & 151549 & 7.863 & 38595 & 9.469 \\
0.05 & 304298 & 15.725 & 75784 & 20.077 \\
\hline
\end{tabular}

The program was written in Visual C++, tested on an Intel i3-4170 CPU at 3.7 GHz, $4 \mathrm{~Gb}$, running under Windows 7. In all the tests, the new version showed the clear and significant preference over the version with linesearch in the total number of computational expenses despite the greater number of iterations. 


\section{CONCLUSION}

In the present work, we proposed a simplified version of the partial linearization method for the network equilibrium problem, in which the stepsize choice is realized without any iterative procedure. This modification maintains the basic convergence properties of the method but enables one to reduce the total volume of computational efforts. The key element of this approach is a given majorant stepsize sequence converging to zero. The next decreased value of the stepsize is taken only when the current iterate does not give a sufficient descent, which is estimated with the help of an Armijo-type condition. Preliminary computational tests showed that such modifications can be more efficient in comparison with the original variant of method because, in particular, the former is more flexible in the choice of parameters. We believe that the proposed approach is promising for further investigations.

\section{ACKNOWLEDGMENT}

The first author was supported by the Russian Foundation for Basic Research (Project 19-01-00431). The results of the first author in this work were obtained within the state assignment of the Ministry of Science and Education of Russia (Project 1.460.2016/1.4). The first and second authors were supported by grant 315471 and 315366 from Academy of Finland.

\section{REFERENCES}

Bertsekas, D.P. et al, 1982. Projection methods for variational inequalities with application to the traffic assignment problem, in Nondifferential and Variational Techniques in Optimization, Springer, Berlin, Heidelberg, pp. 139-159.

Dafermos, S., 1980. Traffic Equilibrium and Variational Inequalities, Transp. Sci., Vol. 14, No. 1, pp. 42-54.

Dafermos, S., 1982. The general multimodal network equilibrium problem with elastic demand, Networks, Vol. 12, No. 1, pp. 57-72.

Konnov, I.V., 2015. On auction equilibrium models with network applications. NETNOMICS: Economic Research and Electronic Networking, Vol. 16, No. 1, pp. 107-125.

Konnov, I.V., 2018a. Conditional gradient method without line-search, Russian Math. (Iz. VUZ), Vol. 62, No. 1, pp. 93-96.

Konnov, I.V., 2018b. Simplified versions of the conditional gradient method. Optimization, Vol. 67, No. 12, pp. $2275-2290$.

Konnov, I. et al, 2016. Partial Linearization Method for Network Equilibrium Problems with Elastic Demands. In: Discrete Optimization and Operations Research. DOOR 2016. Lecture Notes in Computer Science, Vol. 9869, pp. 418-429.

Mine, H. et al, 1981. A minimization method for the sum of a convex function and a continuously differentiable function. J. Optim. Theor. Appl., Vol. 33, pp. 9-23.

Nagurney, A.B., 1984. Comparative tests of multimodal traffic equilibrium methods. Transportation Research Part B: Methodological, Vol. 18, No. 1, pp. 469-485.

Nagurney, A., 1999. Network Economics: A Variational Inequality Approach. Kluwer Acad. Publ., Dordrecht

Patriksson, M., 2015. The Traffic Assignment Problem: Models and Methods. Dover, Mineola, NY

Pinyagina, O.V., 2017. The network equilibrium problem with mixed demand. Journal of Applied and Industrial Mathematics, Vol. 11, No. 4, pp. 554-563. 